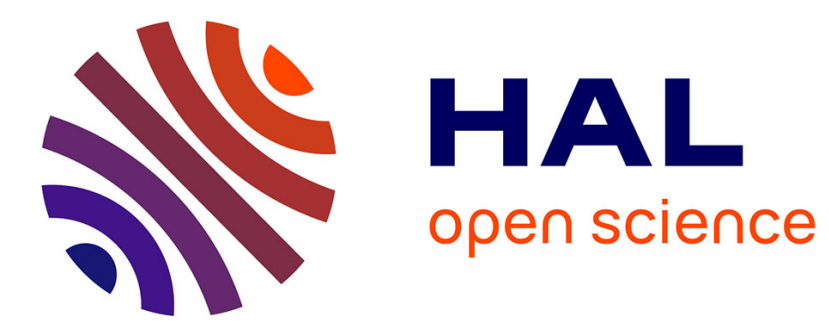

\title{
Réinterroger les sciences sociales à l'heure du numérique. Sociabilité, vie privée et digital labor
}

\author{
Antonio A. Casilli
}

\section{To cite this version:}

Antonio A. Casilli. Réinterroger les sciences sociales à l'heure du numérique. Sociabilité, vie privée et digital labor. XXe Congrès de l'Association Internationale des Sociologues de Langue Française (AISLF), Jul 2016, Montréal, Canada. hal-01625655

\section{HAL Id: hal-01625655 \\ https://hal.science/hal-01625655}

Submitted on 28 Oct 2017

HAL is a multi-disciplinary open access archive for the deposit and dissemination of scientific research documents, whether they are published or not. The documents may come from teaching and research institutions in France or abroad, or from public or private research centers.
L'archive ouverte pluridisciplinaire HAL, est destinée au dépôt et à la diffusion de documents scientifiques de niveau recherche, publiés ou non, émanant des établissements d'enseignement et de recherche français ou étrangers, des laboratoires publics ou privés. 
Réinterroger les sciences sociales à l'heure du numérique.

Sociabilité, vie privée et digital labor

Antonio A. Casilli

Retracer les évolutions récentes des études sociales sur le numérique ainsi que caractériser les débats scientifiques qui agitent ce domaine de recherche signifie identifier des grands attracteurs, des objets d'analyse qui s'imposent par leur urgence et par leur portée, des thématiques qui deviennent le centre focal de controverses. Le geste épistémologiquement significatif qu'il faut accomplir avant tout chose, c'est de rechercher un positionnement par rapport aux différentes approches et courants. Pour nous, le positionnement essentiel consiste dans la mise à distance de la dichotomie latourienne entre analyse des sociétés «déjà faites» et sociétés "en train de se faire $»^{1}$. Si cette approche peut s'avérer utile pour étudier les systèmes techniques des siècles passés, le paradigme actuel des écosystèmes technologiques et des infrastructures nous impose de nous tenir à l'écart de tout dualisme. La société façonnée par le numérique est indubitablement un objet dont la granularité et la fragmentation (géographique, économique, culturelle) rendent visibles des mutations et des dynamiques socio-techniques. Néanmoins elle est « déjà faite » dans la mesure où elle n'est pas seulement le mirage d'une civilisation in fieri mais le croisement de valeurs, de comportements et d'organisations qui sont déjà en existence. Même le côté utopique de la culture numérique s’inscrit résolument dans de schémas politiques contemporains².

L'autre interrogation qui traverse cette contribution concerne évidemment l'impact des technologies numériques sur les domaines du savoir et sur leur structuration au sein des communautés de la recherche. Nous revendiquons depuis plusieurs années une approche «transdisciplinaire dans la discipline $»^{3}$. Cette posture se manifeste dans le choix de nous situer dans un domaine spécifique qui à son tour puise dans plusieurs sciences sociales et sciences exactes, dont il prône en même temps l'ouverture et le renouvellement. Ce sont les digital

\footnotetext{
${ }^{1}$ LATOUR Bruno, Changer la société, refaire de la sociologie, Paris, La Découverte, 2006.

${ }^{2}$ LOVELUCK Benjamin, Réseaux, libertés et contrôle. Une généalogie politique d'internet, Paris, Armand Colin, 2015.

3 CASILli Antonio A., "Pratiquer la transdisciplinarité dans la discipline. Temporalité, territorialité et réalisme des professions scientifiques", in Frédéric Darbellay \& Theres Paulser (dir.) Au miroir des disciplines. Réflexions sur les pratiques d'enseignement et de recherche inter- et transdisciplinaires, Berlin, Peter Lang, 2011, p. 65-81 ; CASILLI Antonio A., "Comment les usages numériques transforment-ils les sciences sociales ? », in Pierre Mounier (dir.), Read/Write Book 2, Marseille: OpenEdition Press, 2012, p. 239-247.
} 
bumanities qui accueillent notre production récente et encadrent notre parcours, ainsi que celui de plusieurs collègues et compagnons de route. Bien que la communauté universitaire ait entrepris une vive discussion portant sur la nature des objets traités (certains insistant sur une matrice « littéraire » et textuelle, d'autres poussant pour l'adoption d'une vision plus inclusive et ouverte à tout phénomène et comportement ayant trait à l'humain), et parfois sur le nom même à donner à la nouvelle discipline dans ses multiples acceptions et déclinaisons (études digitales ? sociologie du numérique ? anthropologie numérique ?) ${ }^{4}$, le débat, à ce stade, cherche avant tout à répertorier les recherches en cours afin de créer des alliances, voire de structurer le domaine de recherche comme un «grand chapiteau » (approche dite de la big tent digital bumanities) ${ }^{5}$.

La question de la nouveauté des méthodes se pose également. La diffusion des technologies de l'information et de la communication dans le monde de la recherche marque l'arrivée et l'utilisation d'outils de plus en plus visibles et performants. L'émergence des big data, l'application de méthodes computationnelles avancées (apprentissage automatique, fouille de grands graphes, systèmes complexes, etc.) à l'étude des mondes sociaux, la création de nouveaux outils au croisement de la sociologie, de l'économie, de la physique et de l'informatique sont autant de nouveaux objets d'études que de manières d'instrumenter nos recherches.

Toutes ces transformations sont cruciales et doivent être prises en compte conjointement. Pour les aborder et pour en montrer des manifestations concrètes, nous procéderons à une caractérisation "par événements", en dégageant trois grandes thématiques, qui sont aussi des grandes controverses ayant agité le domaine des études sociales du numérique ces dernières années. Cela sera pour nous aussi l'occasion de revenir sur nos pas, car ces thématiques ont fait les objets des trois ouvrages que mes co-auteures et moi-même avons publiés entre 2010 et 2015. Ces événements nous aident à tracer un tableau plus complet de la sociologie du numérique, de ses enjeux, de ses courants. Les trois controverses portent alors sur le lien social (surtout ce que les réseaux sociaux numériques font à notre manière de créer des «liens sociaux » pluriels), sur la vie privée (ou plutôt l'équilibre entre vie privée et sphère publique), sur la notion de travail (comment elle n'est pas évacuée, mais transformée, à l'heure des plateformes). Nous allons, pour chacun de ces trois objets, poser la question suivante : comment a-t-il été reconfiguré par le numérique ? Par « reconfigurer", nous entendons, d'une part, "réaménager les forces sociales », organiser de façon originale les acteurs autour et à l'intérieur de ces phénomènes, mais aussi, d'autre part, en reprenant l'acception populaire du verbe (ex. le dépanneur informatique ou l'usager lambda qui configure à nouveau un système d'exploitation quand celui-ci « plante »), celui de bricoler avec les

\footnotetext{
4 CASILLI Antonio A., "Anthropologie et numérique: renouvellement méthodologique ou reconfiguration disciplinaire?", Anthrovision, vol. 1, no. 1, 2014 ; STIEGLER Bernard (dir.), Digital Studies : organologie des savoirs et technologies de la connaissance, Limoges, Fyp éditions, 2014 ; BOULLIER Dominique, Sociologie du numérique, Paris, Armand Colin, 2016.

5 PANNAPACKER William. " "Big Tent Digital Humanities", a View From the Edge, Part 1 », The Chronicle of Higher Education, 31 juillet 2011, http://www.chronicle.com/article/Big-Tent-Digital-Humanities/128434/.
} 
notions héritées de la sociologie traditionnelle, en les questionnant, pour finalement les remettre en fonction.

\section{Le mythe de la « désocialisation numérique»}

Commençons donc par le lien social et ses changements face à l'essor des technologies numériques. Il s'agit de l'un des objets d'étude autour duquel se sont concentrées les plus fortes inquiétudes et manifestées les prises de positions les plus significatives lors de la première vague d'études sur les effets sociaux des technologies de l'information et de la communication. Depuis la dernière décennie du siècle passé, on assiste à la stigmatisation des utilisateurs, accusés de se mettre en retrait face à leurs proches en privilégiant une sociabilité fantasmatique, "virtuelle», non présentielle et centrée sur des liens affinitaires faibles plutôt que sur les solidarités, mais aussi les obligations et les contraintes, propres de leurs milieux sociaux. La caractérisation négative des technologies numériques en tant que vecteurs de "déconnexion sociale ", d'isolement et de pathologies psychologiques multiples (addiction, dépression, violence, etc.), a été basée sur cette représentation dyadique des contextes d'interaction humaine ${ }^{6}$. La vision sous-jacente à cette approche est assez simpliste et, à d'autres occasions, nous l'avons qualifiée de représentation «hydraulique» de la sociabilité en ligne. Deux scènes sociales s'opposeraient, l'une définie « réelle parce que présentielle et matérielle, l'autre conçue comme dématérialisée et médiatisée par les technologies communicantes. Socialité sur internet et sociabilité IRL (In Real Life) sont alors figurées comme deux vases communicants. Si les flux de communication se déplacent trop dans le vase Internet, la vie familiale ou amicale se trouve à $\sec ^{7}$. Le fait de s'inscrire sur un service en ligne aurait donc comme conséquence inévitable l'effondrement de l'univers social présentiel.

L’usage numérique est conçu comme une prise de risque, de perte de contact avec les relations humaines significatives pour la constitution du capital social de tout individu. Néanmoins, si les usagers de la fin du $20^{\text {ème }}$ siècle étaient vus comme des «murés » (selon la définition de Félix Guattari) $^{8}$, ce stéréotype s'estompe avec le web participatif et les usages mobiles du début du $21^{\text {ème }}$ siècle, qui réduisent progressivement l'écart perçu entre la scène sociale médiatisée par les technologies communicantes et la scène présentielle. Le débat au sein les sciences sociales a préparé ce dépassement, en opposant les chercheurs qui se sont efforcés de démontrer que les communications en ligne entraînaient une atomisation sans précédent et ceux qui voyaient dans les formes sociales passant par les communautés online des occasions de coexistence et de construction de rapports interpersonnels viables.

\footnotetext{
6 JURGENSON Nathan «Digital Dualism versus Augmented Reality», Cyborgology, 24 février 2011 https://thesocietypages.org/cyborgology/2011/02/24/digital-dualism-versus-augmented-reality/.

${ }^{7}$ CASILLI Antonio A., Les liaisons numériques. Vers une nouvelle sociabilité ?, Paris, Seuil. 2010.

8 GUATTARI Félix « Communication : Pour une éthique des médias », Le Monde, 6 novembre 1991.
} 
La première posture a été exemplairement incarnée par le psycho-sociologue américain Robert Kraut, porteur de la notion du "paradoxe d'internet »". Suite à son enquête auprès de 73 familles de la ville de Pittsburgh ayant installé une connexion modem depuis moins de deux ans, une (faible) corrélation négative avait été observée entre le fait d'utiliser internet et l'investissement dans la vie sociale présentielle. Cela avait suffi au chercheur pour conclure que cette technologie communicante, au lieu d'aider la cohésion des groupes humains, aurait un effet délétère pour le bien-être psychologique et social des individus concernés. Kraut est revenu sur ces résultats quelques années plus tard pour les désavouer: lors d'une étude de suivi effectuée auprès de la même population, l'effet désocialisant s'avérait inexistant ${ }^{10}$. La raison invoquée par le chercheur était la difficulté de prise en main de ces technologies, dont les équipements n'avaient pas, à la deuxième moitié des années 1990, la vitesse, la performance, l'intuitivité d'usage actuelles. Si l'investissement de temps dans les échanges en ligne des néo-internautes se faisait au détriment des interactions avec leurs liens forts, c'était principalement à cause d'une réallocation de leurs ressources cognitives et de leur temps dispon ible entre s'initier à internet ou communiquer avec leurs proches. La désocialisation numérique était alors un effet transitoire de ces contraintes techniques.

Mais ceci ne suffisait encore à faire admettre aux chercheurs que la sociabilité en ligne se caractérisait par une continuité avec les interactions présentielles. Kraut et son école insistaient pour qualifier les usages informatiques en ligne de «loisirs non sociaux » favorisant l'attitude d'individualisation. Afin de dépasser définitivement le "paradoxe d'internet», il fut nécessaire d'opérer un changement de cap, consistant à resituer les usages au sein des activités productives et au plus près de la coopération humaine. Deux sociologues canadiens, Barry Wellman et Caroline Haythornthwaite, le firent en se plaçant du côté de l'analyse des réseaux sociaux (SNA, Social Network. Analysis) et de la théorie de la multiplexité médiatique (media multiplexity). C'est ainsi que ces chercheurs allaient se doter des outils pour constater empiriquement que, autant du point de vue des interactions professionnelles que de celui des contacts amicaux ou familiaux, les liens numériques multiplient les canaux de communication ${ }^{11}$. Par exemple, des collègues, membres d'une même équipe, ont des besoins et des occasions de communiquer (en face-à-face, par téléphone, par mail, par tchat...) plus importants que des collègues travaillant dans deux départements différents, ou sur deux sites éloignés. Moins de besoins d'échange se traduisent en moins d'occasions d'interaction, en présentiel ou via internet. D'où le constant que ceux qui se servent de technologies d'information et de la communication sur leur lieu de travail affichent un niveau de connexion interpersonnelle plus important avec les membres de leurs milieux

\footnotetext{
9 KRAUT Robert et al. " Internet Paradox: A Social Technology That Reduces Social Involvement and Psychological Well-Being? ", American Psychologist, vol. 53, n. 9, 1998, p. 1017-1031.

${ }^{10}$ KRAUT Robert et al. «Internet Paradox Revisited », Journal of Social Issues, vol. 58, n. 1, 2002, p. 49-74.

11 HAYTHORNTHWAITE Caroline "Strong, Weak, and Latent Ties and the Impact of New Media ", The Information Society, vol. 18, n. 5, 2002, p. 385-401; WELLMAN Barry «Connecting Community: On- and Off-line », Contexts, vol. 3, n. 4, 2004, p. 22-28.
} 
professionnels et ne voient pas diminuer leur temps de socialisation. Les mêmes résultats valent pour les échanges amicaux ou familiaux : un lien «fort» aura plus de canaux de communication (électroniques ou pas), alors qu'un lien faible se cantonnera à des modalités de contact moins «multiplexes » (les inconnus peuvent par exemple se contacter par mail, alors que les membres d'une famille possèdent multiples modalités de contact: par messagerie instantanée, e-mail, téléphone ou en face-à-face). Le web ne remplace pas la communication présentielle, mais il la complète, finissant par augmenter le volume total des interactions entre individus connectés.

La grandeur et la décadence du mythe de la désocialisation d'internet représente le premier événement marquant la sociologie du numérique. Un événement qui montre le potentiel de croissance et de structuration d'une base commune de connaissances au sein de ce champ disciplinaire. Inévitablement, quelques voix continuent de dénoncer les médias numériques comme source d'isolement et de déperdition du lien social ${ }^{12}$, mais le créneau des recherches sur internet et ses sociabilités s'est irréversiblement transformé. Évaluer les conséquences du web et des technologies sœurs sur le lien social ne peut pas se réduire aujourd'hui à examiner les pratiques individuelles. Ce sont les interactions mêmes qu'il convient de prendre en compte. L'analyse des réseaux sociaux, avec ses deux notions-phares de la « force des liens faibles ${ }^{13}$ et de tendance au bridging (c'est-à-dire à combler les trous structurels entre individus ou groupes) ${ }^{14}$, impose ce recadrage analytique amenant à une conception plurielle de la notion de lien social. Les «liens sociaux» peuvent alors être énumérés et évalués autant en ligne qu’en modalité nonmédiatisée. Leur force n'est plus une prérogative des interactions présentielles.

Un exemple tiré d'une recherche récente conduite par l'équipe de Dana Diminescu dans le cadre de l'ambitieux programme TIC-Migrations permet de montrer à quel point l'encastrement entre stratégies relationnelles présentielles et médiatisées par internet est un fait avéré pour les usagers actuels. C'est en analysant les graphes sociaux de jeunes migrants installés en France que l'on constate (fig. 1) que les contacts d'un profil Facebook épousent les structures et les temporalités des relations personnelles façonnées par les études, par la famille, par les institutions, par les trajectoires migratoires ${ }^{15}$.

L'image (a) représente le graphe social extrait d'un profil Facebook d'un étudiant marocain installé à Lille. Chaque nœud de ce réseau est un contact, chaque lien indique que ces contacts sont à leur tour «amis » sur la plateforme sociale. Ces nœuds sont réunis en clusters, c'est-à-dire en groupes plus ou moins denses. L'image (b) illustre le même graphe, mais avec des

12 TURKLE Sherry, Alone Together: Why We Expect More from Technology and Less from Each Other, New York, Basic Books, 2011.

${ }_{13}$ GRANOVETTER Mark, «The Strength of Weak Ties », American Journal of Sociology, vol. 78, n. 6, 1973, p. 13601380.

${ }^{14}$ BURT Ronald, «Structural Holes versus Network Closure as Social Capital», in Nan Lin, Karen Cook, and Id. (dir.) Social Capital: Theory and Research, New York, Aldine De Gruyter, 2001, p. 31-56.

15 MARCHANDISE Sabrina «Investir le web social des étudiants marocains en mobilité internationale. Une méthode imposée par le terrain», working paper e-Diasporas Atlas, 2012 http://www.e-diasporas.fr/workingpapers/Marchandise-MoroccansFacebook-FR.pdf 
commentaires que l'étudiant a tracé lui-même en attribuant à chaque cluster une signification personnelle. L'image (c) est une représentation schématique à partir de ces annotations. La première image nous fournit le point de vue d'un observateur externe aux modes de sociabilité de cet individu (c'est la perspective de la plateforme Facebook ou celle de l'algorithme de visualisation du logiciel d'analyse des réseaux utilisé par les chercheurs). La dernière image, en revanche, nous fournit une interprétation selon le point de vue de l'individu observé, qui après avoir annoté le graphe à la demande des chercheurs, décrit les communautés qui se dégagent et leur donne un sens par rapport à son expérience exclusive. Le graphe social est alors capable de raconter une histoire qui va des écoles primaires, au collège (phase 1), au service militaire (2), à la

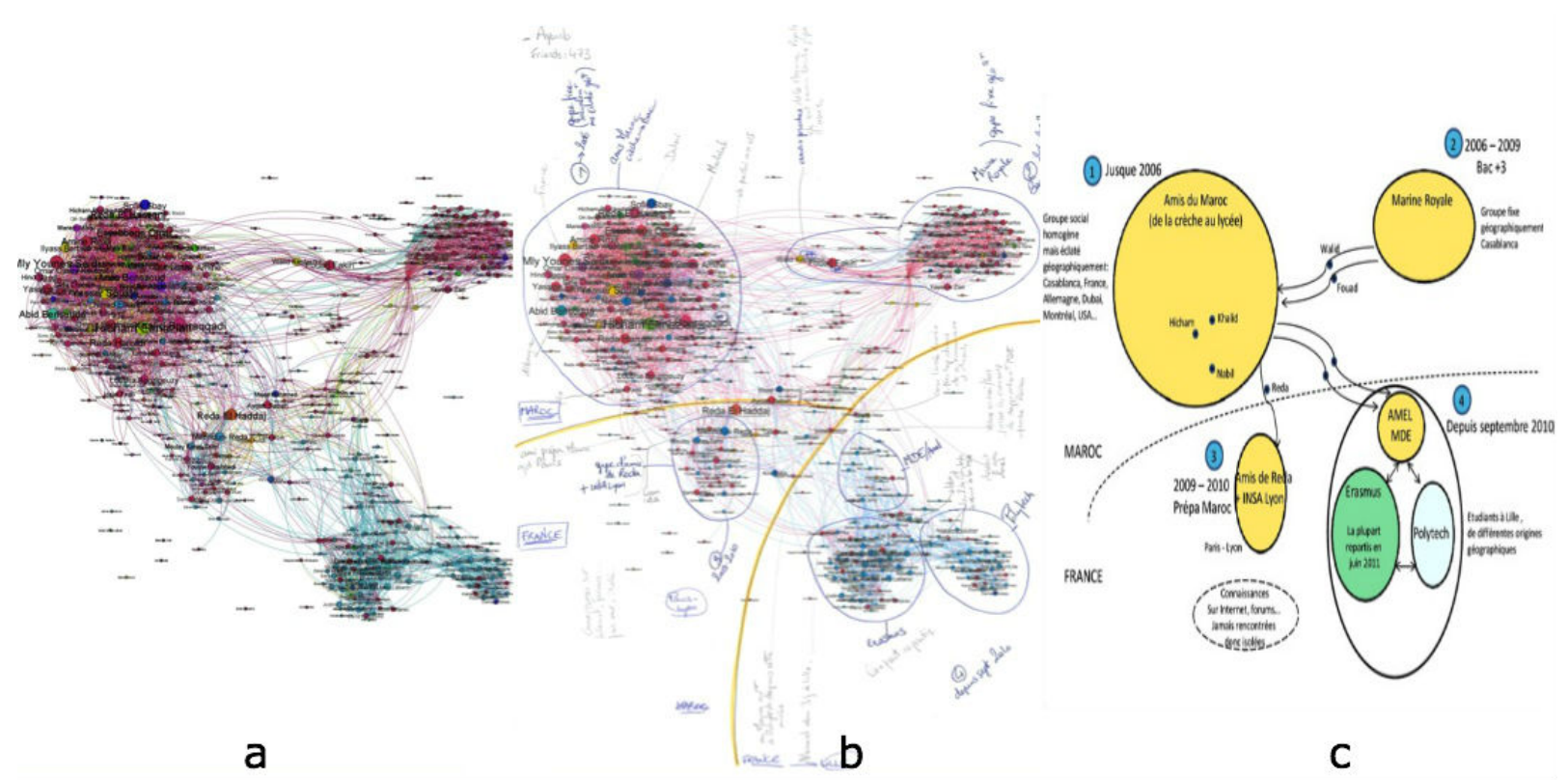

prépa (3) et à l'installation en France (4). Les dates aident à comprendre que chaque phase de ce schéma qualitatif (calqué sur un graphe quantitatif) n'est qu'une époque de l'existence du participant à l'enquête. Le réseau devient, en quelques sorte, un moyen pour livrer un récit de vie dans lequel il n'y a point d'opposition entre « réel » et « virtuel », mais plutôt une continuité autant du point de vue des usagers de technologies numériques, que de celui des chercheurs - pourvu que ces derniers se dotent des méthodes adaptées à l'étude de ces formes sociales.

\section{La vie privée sur internet: une négociation collective sur le marché des données personnelles}

L'époque actuelle se caractérise donc par la tendance à l'encastrement des individus dans des réseaux sociaux dont la formation et le maintien passent par une médiation numérique. Si certains insistent sur les potentialités de cette condition en parlant d'« individualisme en réseau » (networked 
individualism $)^{16}$ et de « société réseau » (network society) ${ }^{17}$, d'autres s'attardent sur les contraintes et les conséquences inattendues en termes d'excès de connexion. Ceci nous permet de passer à la deuxième grande controverse qui a animé les études sociologiques sur le numérique des dernières années: la recherche d'un équilibre entre vie privée et contact avec la sphère publique par l'intermédiaire d'internet et des technologies sœurs. Ce dont il est question ici, c'est d'une part la surexposition de soi sur les réseaux sociaux numériques, de l'autre l'émergence d'un « capitalisme de surveillance » (surveillance capitalism) ${ }^{18}$ où la centralisation du contrôle régalien sur les populations s'articule avec le traçage constant des utilisateurs des grandes plateformes.

Si les années 1990 avaient été dominées par le "paradoxe d'internet», la décennie suivante allait devenir celle du "paradoxe de la vie privée »: des utilisateurs soi-disant soucieux de leur privacy accorderaient délibérément leurs informations personnelles à d'autres internautes et aux administrateurs des services de réseautage en ligne ${ }^{19}$. Mais une analyse plus complète, mettant le déclaratif des utilisateurs en résonance avec le discours commercial des concepteurs des solutions technologiques, pousse à reconnaitre que le débat sur le rôle de la vie privée en réseau s'avère, d'entrée, idéologiquement biaisé. L'hypothèse de la « fin de la vie privée » a été principalement portée par les acteurs du secteur privé, où l'esprit d'entreprise au sens économique du terme se conjugue avec des éléments d'entrepreneuriat moral au sens d'Howard Becker. Un fil rouge unit la déclaration de 1999 du PDG de Sun Microsystems, Scott McNealy (" De toute façon, vous avez zéro vie privée. Tournez la page ! ») et la réunion de la Federal Trade Commission de 2013 où l'« évangéliste en chef » de Google Vinton Cerf avait défini la vie privée comme une « anomalie historique ». Nous assistons à la mise en place d'un discours unitaire, instrumental aux intérêts d'une filière industrielle dont les modèles d'affaires se basent sur l'expropriation de données personnelles et sur leur marchandisation ${ }^{20}$. Cette perspective prend son sens au sein du méta-récit fortement stylisé et politiquement orienté que ces acteurs portent : la transition à la modernité se serait faite au prix de l'abandon de la cohésion sociale des petites communautés locales; l'émergence de la pensée «privée» imposée par la bourgeoisie urbaine serait un distorsion récente qu'internet aiderait à corriger. La nouvelle " norme sociale » (selon une célèbre déclaration publique de Mark Zuckerberg) serait la transparence, la vie en public ${ }^{21}$.

\footnotetext{
${ }^{16}$ RAINIE Lee et WELLMAN Barry, Networked: The New Social Operating System, Cambridge, MA, MIT Press, 2012.

17 CASTELLS Manuel, The Information Age: Economy, Society and Culture. Volume 1: The Rise of the Network Society, Oxford, Wiley Blackwell, 1996.

18 ZUBOFF Shoshana, "Big Other: Surveillance Capitalism and the Prospects of an Information Civilization », Journal of Information Technology, vol. 30, n. 1, 2015, p. 75-89.

19 BARNES, Susan B., "A privacy paradox: Social networking in the United States », First Monday, vol. 11, n. 9,2006 http:// firstmonday.org/ojs/index.php/fm/article/view/1394/1312.

${ }^{20}$ CASILLI Antonio A., "Quatre thèses sur la surveillance numérique de masse et la négociation de la vie privée ", in Jacky Richard \& Laurent Cytermann (dir.), Etude annuelle du Conseil d'Etat "Le numérique et les droits fondamentaux", Paris, La Documentation française, 2014, p. 423-434.

21 ARRINGTON Michael, «Facebook CEO Mark Zuckerberg : TechCrunch Interview At The Crunchies », < https://youtu.be/LoWKGBloMsU >.
} 
En fait cette redéfinition des normes et de la déviance tentée par les entrepreneurs de la Silicon Valley occulte un décalage important entre ce que les plateformes numériques font avec les données personnelles des internautes et les attitudes visibles des utilisateurs, ainsi que leurs réactions face aux agissements des acteurs industriels. Facebook, par son omniprésence sur plusieurs continents et par son ambition d'être un «internet dans l'internet », est bien placé pour être un terrain d'étude idéal. Ce que la firme de Palo Alto a fait les premiers cinq ans de son existence durant, c'est de rentre public par défaut un nombre croissant d'informations personnelles de ses utilisateurs. Photos de profil, adresses email, nom, genre: ces informations sont devenues disponibles non seulement aux «amis», mais aussi à leurs contacts ou à toute personne consultant le média social, y compris les entreprises tierces connectées à l'API du service, les régies publicitaires et les forces de l'ordre qui s'en servent pour collecter des preuves contre des suspects ${ }^{22}$. Cette démarche volontariste de collecte de données et de dévoilement d'informations confidentielles invite à lire les annonces de «fin de la vie privée » comme des discours d'accompagnement. Les utilisateurs, eux, engagent des tactiques d'offuscation (par prolifération de fausses informations), de triche (en créant des pseudonymes et des identités multiples), de dissimulation (avec le recours à des connexions chiffrées et à d'autres outils d'anonymisation), qui visent à protéger la confidentialité des communications.

Une étude menée sur une population d'étudiants américains de l'université Carnegie Mellon (fig. 2), constate que sur la même période concernée par les changements des politiques de confidentialité de Facebook (2005-2011), les utilisateurs avaient décidé de manière massive de passer en privé (bleu) des informations qui auparavant étaient disponibles en public par défaut (rouge) : études, lieu et date de naissance, goûts musicaux, cinématographiques, littéraires... ${ }^{23} \mathrm{Si}$, d'un côté, l'entreprise force ses usagers à ouvrir leurs données personnelles, de l'autre les utilisateurs contrent cette tendance en se soustrayant autant que possible au regard de leurs pairs et des data miners d'internet.

\footnotetext{
22 SOGHOIAN Christopher, The Spies We Trust : Third Party Service Providers and Law Enforcement Surveillance, thèse de doctorat soutenue à l'Indiana University, 2012.

${ }^{23}$ STUTZMAN Fred, GROSS Ralph, ACQUISTI Alessandro, «Silent Listeners: The Evolution of Privacy and Disclosure on Facebook », Journal of Privacy and Condentiality, vol. 4, n. 2, 2012, p. 7-41.
} 


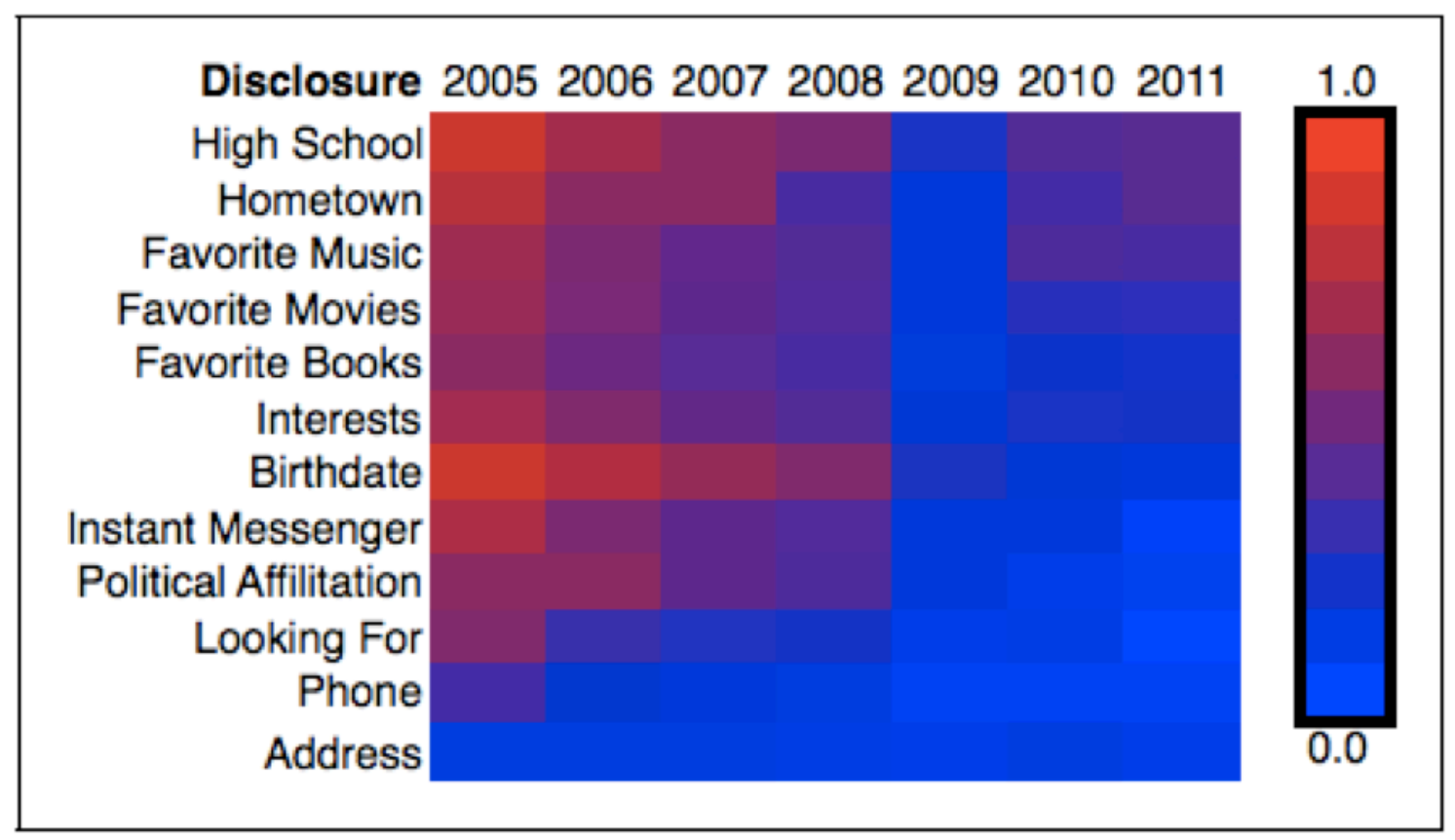

Figure 2 - Les utilisateurs de Facebook passent en privé (bleu) des informations personnelles qui auparavant étaient en public par défaut (rouge). Source : Stutzman et al. 2012.

Comment expliquer cette contre-dynamique ? Jusqu’à quel degré est-elle généralisable ? Pour répondre à ces questions, mes co-auteures, Paola Tubaro et Yasaman Sarabi, et moi-même avons utilisé une méthodologie mixte, conjuguant des modélisations mathématiques et des données qualitatives. Cette méthode «ethno-computationnelle $»^{24}$ se base sur un algorithme qui reproduit les interactions des personnes interagissant sur un média social, codé à partir d'un ensemble de résultats d'enquêtes précédentes. L'aboutissement de la démarche est un modèle multi-agents, c'est-à-dire une simulation informatique de l'évolution des graphes sociaux des internautes. En poussant à l'extrême la double dynamique de surexposition imposée par les plateformes et de protection des données personnelles mise en place par les utilisateurs, un nombre fini de scénarios possibles se dégage. Nous avons pu suivre de millions d'interactions d'agents artificiels, depuis leur inscription sur un média social jusqu'à la présentation de soi et à la création d'une liste de contacts. Au fur et à mesure que ces agents entraient en contact avec d'autres membres du réseau social numérique, ils exprimaient leurs préférences et opinions et recevaient des feedbacks positifs ou négatifs qui les aidaient à mettre au point leur profil et leur style de présentation. En moyenne, davantage d'ouverture aux autres impliquait la création d'un nombre plus important de liens, ce qui conduisait à la création de clusters denses - qui évoquent la représentation populaire des «bulles de filtre $»^{25}$. Mais dans ces mini-communautés où la pression sociale devenait plus tangible, le besoin de protéger sa vie privée se faisait sentir de manière croissante. Si, au moment de sa première inscription, l'utilisateur lambda avait couramment fourni des informations sur lui-

\footnotetext{
${ }^{24}$ TUBARO Paola et CASILLI Antonio A., «'An ethnographic seduction': how qualitative research and agent-based models can benefit each other », Bulletin de Méthodologie Sociologique, vol. 106, n. 1, 2010, p. 59-74.

${ }^{25}$ PARISER Eli, The Filter Bubble: What The Internet Is Hiding From You, New York, Penguin Press Limited, 2011
} 
même, une fois sa communauté formée, il redécouvrait un réflexe vers l'occultation de ses contenus et ses opinions.

Si cette évolution paraît spontanée, c'est qu'elle découle des choix des individus. Mais se limiter à ce niveau micro et méso-social d'analyse c'est faire fi des dynamiques macro-sociales provoquées par les politiques des données développées par les propriétaires des plateformes. Ces derniers ont intérêt à que les utilisateurs ne protègent pas leur vie privée. Ils mettent constamment en place des mesures dissuasives : des interfaces visant à enfouir les fonctionnalités de protection de la confidentialité ; des discours stigmatisant qui assimilent la vie privée au fait d'avoir «quelque chose à cacher»; etc. Quand ces mesures échouent, c'est par une approche de force brute que le partage et l'ouverture des données se fait: du jour au lendemain la firme implante une nouvelle fonctionnalité ou introduit une nouvelle règle dans ses conditions générales d'utilisation, pour "déverrouiller» les informations que les utilisateurs protègent jalousement.

La figure 3 illustre ce que nous venons d'exposer: après un ajustement initial (a) où les utilisateurs laissent baisser leur niveau moyen de privacy pour développer leur communauté (b), ils dérogent au partage de leurs informations et reviennent à des interaction privées (c). Les interventions exogènes de la part des propriétaires des plateformes (qui repassent les données personnelles des utilisateurs en "public par défaut»), conjuguées avec le tendanance des internautes à la protection, engendrent alors des « cycles de privacy » $(\mathrm{d})^{26}$.

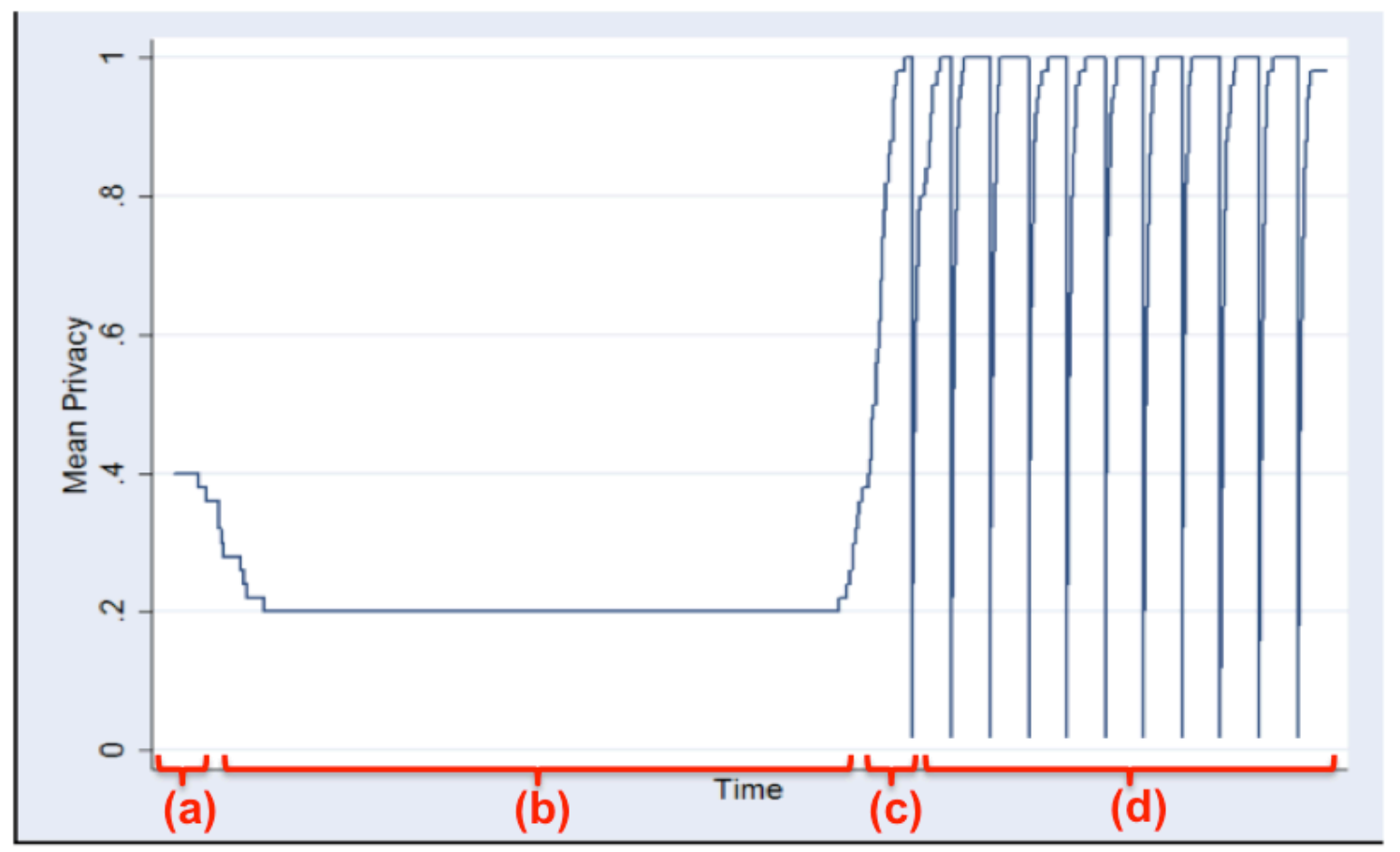

Figure 3 - Cycles de privacy sur les médias sociaux. Source : Tubaro et al. 2012.

\footnotetext{
${ }^{26}$ TUBARO Paola, CASILLI Antonio A., SARABI Yasaman, Against the bypothesis of the "end of privacy". An agent-based
} modelling approach to social media, New York, Springer, 2014. 
Malgré la rhétorique ambiante, la prédisposition des internautes au partage et la dérive politique vers un monde ou la valeur démocratique de la vie privée cède le pas à la nouvelle conception de "publicité par défaut», sont tout sauf avérées. Au contraire, l'expérience sociale sur les plateformes numériques est une dialectique conflictuelle, une négociation collective entre société civile, entreprises du secteur technologique et décideurs publics. Cette négociation ne ressemble en rien à l'évolution apaisée décrite par les théoriciens du « paradoxe de la vie privée ». Au contraire, elle affiche tous les traits d'une véritable guerre culturelle, dont les batailles sont déclenchées par des changements cycliques des politiques de confidentialité. Pour Facebook, ces changements vont du choix de créer en 2006 sa fonctionnalité la plus iconique (le «mur» où s'affichent messages et mises à jour des contacts) à l'acceptation de la décision de la TRAI, le gendarme indien des télécommunications qui, en 2016, a interdit la distribution commerciale du service Free Basics à des centaines de millions d'internautes. Entre les deux, un tournant majeur est marqué par les révélations de 2013 d'Edward Snowden sur le rôle des médias sociaux dans la surveillance de masse de la NSA et de la GCHQ. Le tableau 1 résume ces événements : chaque ligne représente un «incident de vie privée ». La première colonne détaille les dates, la deuxième décrit succinctement l'incident, la troisième colonne détaille les réactions des utilisateurs et des pouvoirs publics, la dernière les conséquences pour l'entreprise. 


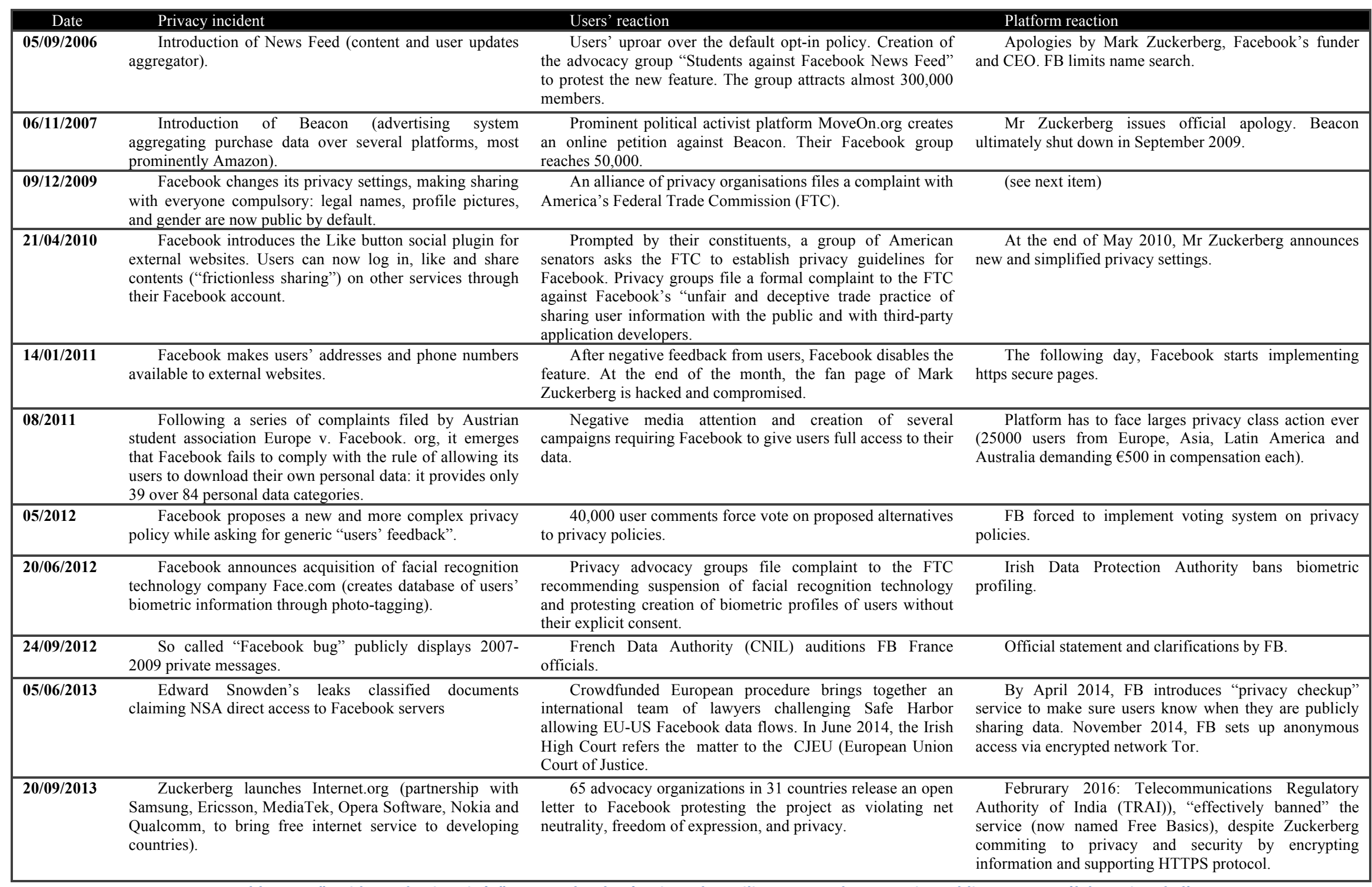

Tableau 1 - "Incidents de vie privée" sur Facebook, réactions des utilisateurs et des pouvoirs publics. Source : élaboration de l'auteur. 
Face aux pressions de l'entreprise Facebook vers la transparence des données personnelles des utilisateurs, ces derniers sont tout sauf passifs : ils réagissent, protestent et, au fil des années, s'organisent en arrivant à se coordonner avec des institutions comme la Federal Trade Commission aux États-Unis ou la CNIL en Europe.

\section{Où est passé le travail à l'heure des plateformes ? La question du digital labor}

Malgré les formules à sensation, la vie privée est encore là, au centre même de notre débat politique, douloureusement au cœur de nos préoccupations quotidiennes. Cela permet aussi de passer à la troisième et dernière thématique débattue dans la sociologie du numérique, qui se concentre sur un aspect particulier de la frontière entre privé et public, à savoir l'arbitrage entre vie personnelle et travail. La production et la valorisation de données personnelles deviennent la base de l'économie des plateformes ${ }^{27}$, surtout depuis la généralisation des usages mobiles et l'éclosion de l'internet des objets, alliant domotique, bracelets connectés et technologies d'autoquantification (quantified self). Pour les possesseurs de ces équipements, les repas ou le sommeil mêmes, et plus en général toute activité nécessaire à la reproduction de la force de travail, n'est plus à l'écart de la production de valeur. Chaque instant de la vie connecté devient une occasion pour créer de données par un clic, par une communication, même par le simple fait d'être connecté.

Une première conséquence de ces transformations est le brouillage des frontières entre travail et loisirs. Si ces activités étaient auparavant distinctes, elles sont désormais mélangées dans un seul cycle de production qui ne s'arrête jamais. La recherche en sciences sociales des dernières années propose de les associer par le biais de nouveaux concepts tels que weisure (work + leisure) ${ }^{28}$ ou playbor (play + labor $)^{29}$. Cette modalité d'extraction de valeur des usages numériques remet en question la notion même de «temps de travail ». L'ancienne devise du syndicalisme du XIX ${ }^{\text {ème }}$ siècle (« nous voulons 8 heures pour travailler, 8 heures pour nos loisirs, 8 heures pour reposer ») serait désormais remplacée par 3 fois 24 heures de travail/loisir/repos indifférencié et équipé par nos outils connectés.

Les usages numériques ubiquitaires se situent aux antipodes des « loisirs non sociaux » dont parlait Robert Kraut (v. supra). La question est, à l'inverse, que cette utilisation intensive arrive à pénétrer les interstices de l'activité humaine en inscrivant potentiellement toute activité sociale dans un contexte de production de valeur marchande. C'est là une façon de circonscrire le périmètre de la dernière controverse qui agite les sciences sociales à l'heure d'internet: celle relative à la transformation des usages numériques en digital labor, c'est à dire en un ensemble de tâches fragmentées, déqualifiées, gratuites (ou faiblement rémunérées), qui constituent le «travail

\footnotetext{
27 SPIEKERMANN Sarah, BÖHME Rainer, ACQUISTI Alessandro, HUI Kai-Lung, "Personal data markets », Electronic Markets, vol. 25, n. 2, 2015.

${ }^{28}$ Conley, D. (2008). Elsewhere, U.S.A.: How We Got from the Company Man, Family Dinners, and the Affluent Society to the Home Office, BlackBerry Moms, and Economic Anxiety, New York: Pantheon.

${ }^{29}$ Kücklich, J. (2005). Precarious Playbour: Modders and the Digital Games Industry. Fibreculture Journal, 25(1).
} 
implicite » des usagers des plateformes. Le débat, qu'avec mon collègue Dominique Cardon nous avons résumé dans un ouvrage à deux voix ${ }^{30}$, porte justement sur le dilemme de qualifier ces usages numériques comme de la participation spontanée et «capacitante » ou bien comme des formes de captation de la valeur produite par les utilisateurs, faisant l'objet de luttes pour leur reconnaissance.

Assimiler tout usage à du travail serait en effet une provocation, ou du moins une manière un peu hâtive d'introduire les termes de ce débat. Assurément, un nombre croissant de dispositifs technologiques s'appuie sur les contributions plus ou moins gratuites de leurs usagers: des contenus générés par les utilisateurs des plateformes multimédias comme Youtube ou Instagram, au crowdworking des foules qui réalisent le bouche à oreille nécessaire pour le marketing viral, jusqu'au petits jobs des utilisateurs des plateformes collaboratives. Des formulations populaires (comme par exemple «Poster sur Facebook, c'est travailler $»^{31}$ ), quoique réductrices, aident à synthétiser l'enjeu de l'assimilabilité de toute activité médiatisée par le numérique à un geste productif qui engendre des bénéfices (économie des données), qui est soumis à des contraintes contractuelles (conditions d'utilisation des plateformes), qui est mesuré et enregistré (par les compteurs, les scores et les nombre de partages qui adoubent les profils sur les médias sociaux). Pour autant que les activités métrologiques sont indissociables de l'attribution de qualités à un produit ou à un service, un simple « like » devient un geste de valuation à part entière ${ }^{32}$.

Inscrire les usages numériques dans le temps de vie des internautes signifie alors accompagner la détection d'un changement du ratio entre le travail formel (celui qui est encadré par des contrats d'emploi, visible en tant qu'activité productive dont les droits sont reconnus, borné à un endroit et à un horaire) et le travail informel (qui peine à être reconnu en tant que tel et qui est constamment qualifié d'autres termes: partage, participation, collaboration, etc). Les relations entre individus (ou entre individus et communautés) passent aujourd'hui par la médiation numérique, qui met un place une civilisation du «travail implicite» qui se manifeste par les contributions en ligne, par l'attention à des flux d'information, par la curation de contenus. Cela jette une lumière nouvelle sur la notion de la «fin du travail», qui fait l'objet d'une vive contestation depuis deux décennies. Non seulement, comme d'autres l'avaient déjà bien souligné, le travail demeure générateur de relations humaines ${ }^{33}$, mais sa prétendue disparition à l'heure d'internet (tout comme celle de la vie privée ou du lien social auparavant) n'est que l'effet d'un double aveuglement : le refus de reconnaître les transformations des modes de sociabilité et celui de détecter les adaptations du capitalisme dans le contexte des plateformes numériques. En particulier, une certaine sociologie des usages n'a voulu voire dans la présence sur internet des

\footnotetext{
${ }^{30}$ CARDON Dominique et CASILLI Antonio A., Qu'est-ce que le digital labor?, Paris, Ina Éditions, 2015.

31 FERAUD Jean-Christophe «Poster sur Facebook, c'est travailler. Comment nous rémunérer ?», Libération, 11 septembre 2015

32 CALLON Michel, «Postface : La formulation marchande des biens », in François Vatin (dir.), Evaluer et valoriser : une sociologie économique de la mesure, Toulouse, Presses Universitaires du Mirail, p.247-269, 2009.

${ }^{3}$ MEDA Dominique, Le travail. Une valeur en voie de disparition?, Paris, Aubier, 1995.
} 
individus contemporains qu'une expression de la consommation ostentatoire (conspicuous consumption). Nous pouvons au contraire y voire le modèle d'une production discrète (inconspicuous production $)^{34}$ où l'envie de participation et de générosité est indissociable d'un certain degré d'exploitation économique.

L'émergence des conflictualités spécifiques du travail des plateformes attire l'attention des chercheurs. Les « amateurs » et les contributeurs «bénévoles » d'internet se fédèrent désormais en syndicats, en associations. Ils organisent des actions concertées pour faire venir à la surface des revendications distinctives. Nous assistons alors au refus des usagers des plateformes à la demande d'être relégués dans l'économie «collaborative » et à leur lutte pour être requalifiés en salariés (v. les controverses autour des chauffeurs Uber). Mais ce sont aussi les litiges individuels sur les conditions contractuelles des créateurs de contenus multimédias (sur Youtube, Huffington Post, etc.) qui se multiplient. Même les administrateurs des forum de discussion s’invitent dans les luttes des salariés et créent des nouvelles solidarités ( $\mathrm{v}$. la grève des modérateurs de Reddit en 2015), alors que les utilisateurs de Google et de Facebook lancent désormais des recours collectifs pour que leur «travail invisible de producteurs de données» soit reconnu (v. par ex. la class action Massachussetts contre Google ReCaptcha aux États-Unis, ou celle de l'association Europe v. Facebook devant la Cour de Justice de l’Union Européenne).

Les utilisateurs qui revendiquent un rôle de travailleurs des plateformes nous mettent face à un travail de type nouveau, qui ne s'appuie plus sur les critères classiques de pénibilité, subordination, rémunération. En particulier, le facteur de la rémunération monétaire devient caduc. Le digital labor est inscrit sur un continuum entre activités rémunérées (c'est le cas des l'économie à la demande), micro-rémunérées (comme pour les «tâcherons du clic» des plateformes de micro-travail), ou non rémunérées (comme pour certaines plateformes sociales).

Cette affirmation mérite d'être développée. Commençons par la dernière partie : la gratuité des réseaux sociaux numériques n'est pas une fatalité, mais l'effet de choix très précis en termes de modèles d'affaires. Certes, Facebook permet à ses usagers de publier leurs contenus et d'entretenir leurs relations sociales sans contrepartie ${ }^{35}$. Mais le réseau de Mark Zuckerberg ne verse pas non plus des dividendes ou des salaires aux utilisateurs dont les données sont monétisées. Le public présume qu'entre la plateforme et ses usagers il n'y a pas d'échange monétaire. En revanche, d'autres plateformes sont basées sur un modèle d'affaires dissemblable. Afin d'attirer des nouveaux membres, non seulement elles proposent leur service à prix zéro, mais arrivent jusqu’à offrir une rétribution en échange de la présence sur le service (c'est ce que les économistes appelleraient un "prix négatif»). Un exemple parmi d'autre peut être la plateforme américaine Tsu. Avant de se frotter à la concurrence impitoyable du géant de Palo

\footnotetext{
${ }^{34}$ Le terme avait été introduit par LIEBERMAN Jethro K., The tyranny of the experts;: How professionals are closing the open society, New York, Walker, 1970.

35 Des exceptions significatives sont représentées par les posts sponsorisés (pour lesquels les utilisateurs paient à la plateforme un tarif proportionnel au nombre de lecteurs potentiels).
} 
Alto et de fermer en 2016, Tsu proposait une idée commerciale fondée sur la transparence : la plateforme admettait vendre les données extraites des profils de ses utilisateurs, mais ces derniers recevaient un pourcentage des revenus éventuels. Après avoir prélevé une commission de 10\%, Tsu versait $45 \%$ de ses recettes au propriétaire du profil $\mathrm{X}$, puis $30 \%$ à la personne $\mathrm{Y}$ qui avait invité le $\mathrm{X}$ à rejoindre le réseau, puis $10 \%$ à $\mathrm{Z}$ qui avait invité $\mathrm{Y}$, et ainsi de suite jusqu'à compenser à peu près tous les membres du service.

Ce modèle de rémunération capillaire permet de distinguer, derrière la soi-disant gratuité des plateformes, une valorisation marchande de données que pour les entreprises du secteur numérique est indissociable de l'activité productive des usagers. La "gratuité » est un concept dont l'application n'est limitée qu'à certaines plateformes, et plus précisément à certaines catégories d'internautes. Même sur Facebook, les utilisateurs vérifiés et d'autres professionnels peuvent monétiser leurs publications. De surcroit, une vaste économie souterraine de clics englobe les échanges «organiques» et le partage spontané d'information. Certains services permettent d'acheter des «likes » et des contacts. Ce sont les fermes à clics, des entreprises qui peuvent augmenter artificiellement le nombre d'abonnés à une page ou faire monter les chiffres des téléchargements ou les notes d'un contenu multimédia. Ces clics sont réalisés dans la plupart des cas par des ressortissants de pays en voie de développement, régulièrement inscrits sur la plateforme, qui sont payés à la pièce pour «aimer» des contenus sur lesquels ils n'ont pas de contrôle. Ils sont compensés est à la hauteur de quelques centimes. Dans des pays caractérisés par des forts taux de chômage et de faibles salaires, ceci peut constituer une alternative viable à la précarité.

Derrière la viralité des contenus, derrière l'effervescence des partages et la richesse des échanges se cacherait alors un monde submergé, fait de conditions de travail souvent déplorables, de volatilité des rémunérations, où le travail est invisible parce que délocalisé à l'autre bout du monde. Une étude récente de l'Oxford Internet Institute ${ }^{36}$ montre l'existence d'importants flux de micro-transactions entre les pays du Sud, devenus producteurs de clics, et les pays du Nord, devenus acheteurs de données. Aujourd'hui, les plus grands réalisateurs de micro-tâches se trouvent aux Philippines, au Pakistan, en Inde, au Népal, à Hong-Kong. Les principaux acheteurs sont aux États-Unis, au Canada, en Australie et au Royaume-Uni (fig. 4).

\footnotetext{
${ }^{36}$ LEHDONVIRTA Vili, BARNARD Helena, GRAHAM Mark, HJORTH Isis, « Online labour markets - levelling the playing field for international service markets? », communication présentée au colloque IPP2014: Crowdsourcing for Politics and Policy, University of Oxford, 2014.
} 


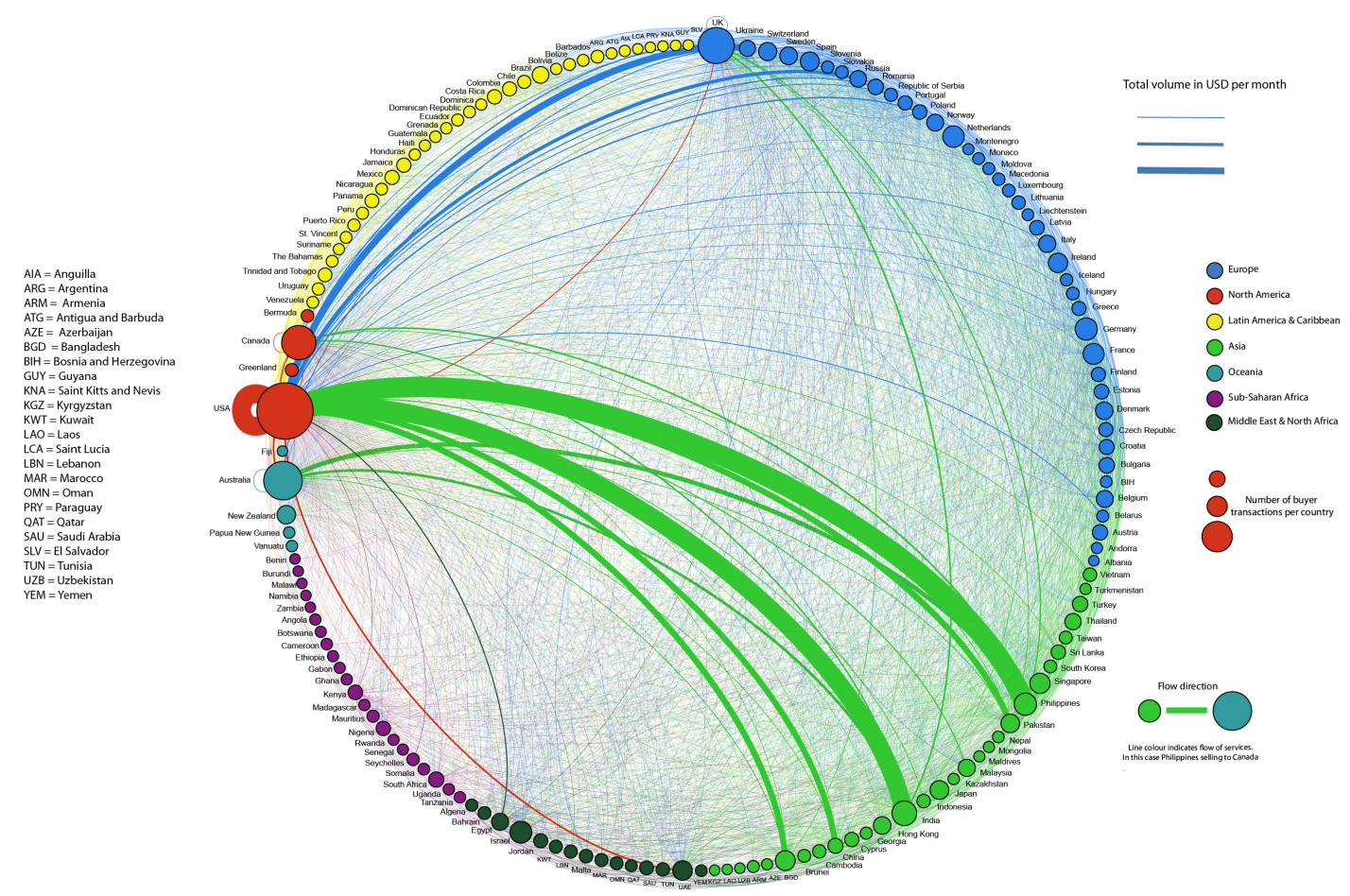

Figure 4 - Micro-travailleurs d'Asie, et recruteurs en Europe, Australie et Amérique du Nord sur une plateforme de digital labor.

Les inégalités classiques Nord/Sud se reproduisent à une échelle considérable. D'autant qu'il ne s'agit pas d'un phénomène résiduel, mais d'un véritable marché du travail : une plateforme de micro-travail comme UpWork compte plus de 12 millions d'utilisateurs, Freelancers a dépassé les 20 millions, et les géants chinois comme Witmart ou Tasken affichent respectivement 7 et 10 millions de micro-ouvriers numériques. Par comparaison, le service Amazon Mechanical Turk (spécialisé dans la réalisation de tâches de retranscription, labélisation d'images ou production de petits commentaires nécessaires pour entraîner les intelligences artificielles) n'est qu'un lilliputien : à peine 500000 utilisateurs, répartis entre les États-Unis et l'Inde.

Par delà ces chiffres, qui sont sujets à caution en l'absence de métriques cohérentes de l'impact économique des plateformes de micro-travail, les exemples que nous venons de présenter s'inscrivent dans des dynamiques plus larges. L'économie du clic a des conséquences pour tous les citoyens d'une société connectée, pas seulement pour ceux qui vivent dans des situations de précarité dans des pays en voie de développement. Dans la mesure où n'importe quel usage numérique, du plus ludique et libre au plus contraint et exploité, peut être capté par des plateformes à but lucratif, nos échanges et relations sociales médiatisés par internet s'articulent avec une forme de travail décentralisé, atomisée et flexible dont les enjeux sont importants et inquiétants pour l'avenir de nos sociétés ${ }^{37}$.

\footnotetext{
37 CASILLI Antonio A., «Is There a Global Digital Labor Culture? : Marginalization of Work, Global Inequalities, and Coloniality», communication dans le 2e symposium du Project for Advanced Research in Global Communication (PARGC), avril 2016, Philadelphie, Etats-Unis <https://hal-mines-paristech.archivesouvertes.fr/halshs-01387649v1>.
} 


\section{Le numérique pour mettre en visibilité les mondes sociaux}

Dans les pages qui précèdent, nous nous sommes donné pour but de passer en revue trois importants moments dialectiques qui ont donné forme aux études sociales du numérique des dernières années. La recherche désormais n'envisage pas les technologies de l'information et de la communication comme un domaine d'activité à part, mais plutôt comme un écosystème à l'intérieur duquel les processus sociaux et les milieux technologiques sont indissociables.

Pour l'étudier, les catégories propres des sciences sociales ont besoin d'être reconfigurées. Cela est nécessaire afin de résoudre la panne épistémique qui consiste à ne voir que la «fin» ou la «mort» de nos objets d'étude-comme c'était le cas pour les trois thématiques que nous avons abordé ici. Les modalités de socialisation, la recherche de l'équilibre public/privé, les institutions du travail ont certainement été ébranlées par l'arrivée du numérique, mais elles ne peuvent pas être déclarés disparues. Leur recomposition dans des cadres analytiques originaux s'impose.

Cette courte incursion dans les digital humanities a permis de déplacer le regard dans la direction de «nouveaux mondes» sociaux dont la nouveauté principale est une forte continuité avec les dimensions ordinaires du vivre ensemble. Le lien social continue d'être la force qui dépasse les différences humaines et les écarts différentiels à la norme. La vie privée continue d'être le droit fondamental qui s'érige contre les abus et qui offre l'opportunité de s'autodéterminer face à la pression vers la conformité culturelle. Le travail continue d'être une galaxie de conflits et de désidératas collectifs dont la dimension politique ne peut pas être éludée. Trois objets, donc, qu’à un moment la communauté scientifique et le débat publique ont a cru anéantis par internet, mais qui restent à leur place.

Par delà les convoitises de «ré-enchanter le monde » face à la civilisation numérique ${ }^{38}$, nous prônons plutôt un effort pour mettre en visibilité les mondes sociaux que ces objets convoquent. Comment est-elle structurée, comment fonctionne-t-elle, la société médiatisée par internet? A quelles subjectivités donne-t-ils lieu ses liens sociaux pluriels, sa vie privée devenue négociation collective, son travail « implicite » et hybridé avec le loisir?

Nous l'avons vu : répondre à ces questions demande un ajustement de nos méthodologies et un décloisonnement disciplinaire. Le renouveau des disciplines, ou leur assemblage dans des champs du savoir naissants, traduit les changements des mondes sociaux et compose avec la multitudes des acteurs sociaux qui les habitent. Les outils à notre disposition constituent d'excellentes opportunités d'intégrer des espaces jusqu'ici extraordinairement difficiles à atteindre en notre qualité d'enquêteurs. Les échanges intimes, les segmentations les plus fines des activités, les motivations en train de se révéler peuvent être enregistrées à partir de traces en ligne et

38 STIEGLER Bernard, Réenchanter le monde: La valeur esprit contre le populisme industriel, Paris, Flammarion, 2008. 
analysées grâce aux nouvelles méthodes d'exploration, de mesure et de modélisation. Mais il ne serait pas prudent de céder la fascination pour ces nouveaux outils sans questionner les procédés et les logiques qui les régissent. C'est pourquoi, les chercheurs en sciences sociales aujourd'hui doivent s'ouvrir au dialogue avec les sciences exactes et celles de l'ingénieur, pour pouvoir avoir une confiance éclairée et raisonnée dans les méthodes qu’ils emploient. Il sera alors question de développer des compétences et des réflexes de pensée originaux. 